\title{
Nozzle based compact spinning
}

\begin{abstract}
Compact spinning is one of the most successful innovations in the 21 st century in spinning industry. Today, the available dominant compact spinning systems are the pneumatic compact spinning systems, which adopt perforated drum, perforated apron or lattice apron as condensing elements. In this paper a novel pneumatic compact spinning is proposed, that is, a precisely designed nozzle as the condensing element and a holding roller were added on the conventional ring spinning frame. When a conventionally drafted roving strand passes by the front nip, it will immediately enter the cavity of the nozzle and is affected by the inside vortex, which finally resulted into a partially wrapped and thinner round structure. The structure of the fiber strands delivered from the holding roller was viewed and recorded through the consecutive pictures. The 4 cases of wrapping structure were statistical analyzed. The results show that more than $1 / 4$ strands are in wrapped structure. The width of the fiber strand passing by the nozzle was measured, which is significantly narrowed, compared with the fiber strand before entering the nozzle. According to the principle of compact spinning, a viable yarn with less hairiness may be produced from this system.
\end{abstract}

Volume 4 Issue 2 - 2018

\author{
Keyi Wang, ${ }^{1,2}$ Wenlian Xue, ${ }^{1,2}$ Longdi Cheng ${ }^{1,2}$ \\ 'Key Laboratory of Textile Science \& Technology, Ministry of \\ Education, Donghua University, China \\ ${ }^{2}$ College of Textiles, Donghua University, China
}

Correspondence: Longdi Cheng, College of Textiles, Donghua University, Shanghai 201620, China, Tel ++86-21-67792689,

Email Idch@dhu.edu.cn

Received: February 22, 2018 | Published: March 30, 2018

Keywords: compact spinning, vortex, modification, nozzle

\section{Introduction}

Compact spinning is regarded as one of the most successful innovations in the $21^{\text {st }}$ century, which can eliminate the spinning triangle and make almost all fibers incorporated into the yarn structure under the same tension. The resulted yarns have less hairiness, and better strength as well as elongation properties, compared with conventional ring spun yarns..$^{1-4}$

There are three types of compact spinning system on the market now: pneumatic system, mechanical system and mechanicalpneumatic system. The pneumatic system is the dominant one, which adopts perforated drum, perforated apron or lattice apron as condensing elements. ${ }^{5,6}$ The roving strand emerging from the front roller nip is condensed by negative pressure airflow produced by the aforementioned elements. The air current is running in open space, which caused high additional energy consumption during spinning process. $^{6}$

In this paper, a novel compact spinning method is reported. A conventional ring spinning spintester was modified by adding a precisely designed nozzle as the condensing element between the front roller and twist insertion. The widths of the fiber strand before entering the nozzle and passing by the nozzle are measured. The yarn formation principle and morphology of the fiber strand from holding roller are discussed.

\section{Yarn formation principle}

As shown in Figure 1, a precisely designed nozzle and a holding roller are added to the conventional ring spinning system. The inlet of the nozzle is inserted to the nip line of front roller. The distance between nips of front roller and holding roller is smaller than the average length of the fibers to be spun, so the drafted fibers could be firmly held when they are running from front roller to holding roller.

In this novel pneumatic compact spinning, the pre-twister is located very closely to front roller nip. After draft of roving, the strand of fibers from the nip of front roller is fed into the pre-twister where the air is compressed from the 4 nozzles, as shown in Figure 2.

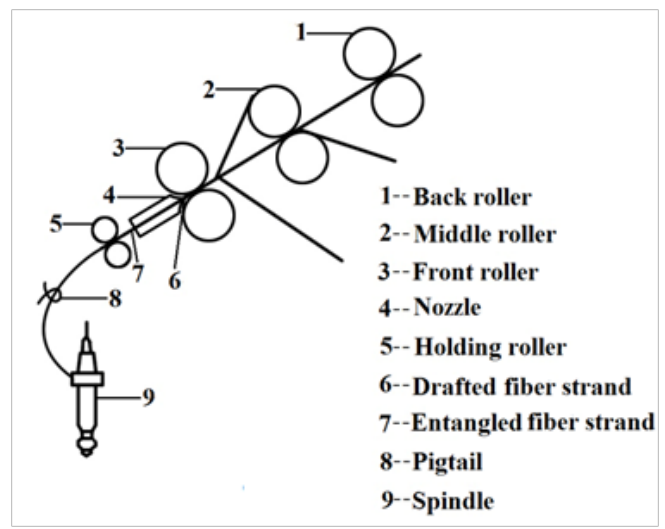

Figure I Schematic diagram of the modified ring spinning system

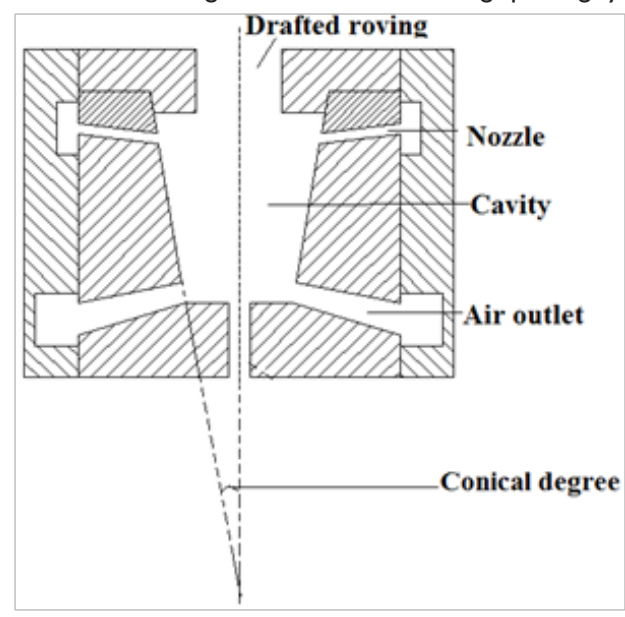

Figure 2 Cross-sectional view of the pre-twister 
The vortex in the nozzle is spiral, which is tangential to the inner wall of the round cavity, flow downwards toward the holding roller. The axis area in the nozzle is not affected by the airflow, while the rest radial area from inlet to outlet is affected strongly. After being drafted in the draft zone, the fiber strand is immediately fed into the nozzle where the vortex exists. The fibers emerged from the nip line of the front roller are parallel and can be classified as edge fibers and core fibers. The leading ends of edge fibers (called free leading ends) are sucked into the nozzle by the vortex while another ends are firmly held by the front roller, as shown in Figure 3. At the same time, both ends of the core fibers are held by the front roller and holding roller respectively. The free leading ends would be wrapped on core fibers by spiral airflow force. With the entanglements formed by the free leading ends, which are controlled by the holding roller continuously, the vortex converts the drafted fiber strand into a condensed and entangled structure. According to the principle of compact spinning, ${ }^{7}$ it then could be positively twisted into a novel yarn with less hairiness by ring and traveler.

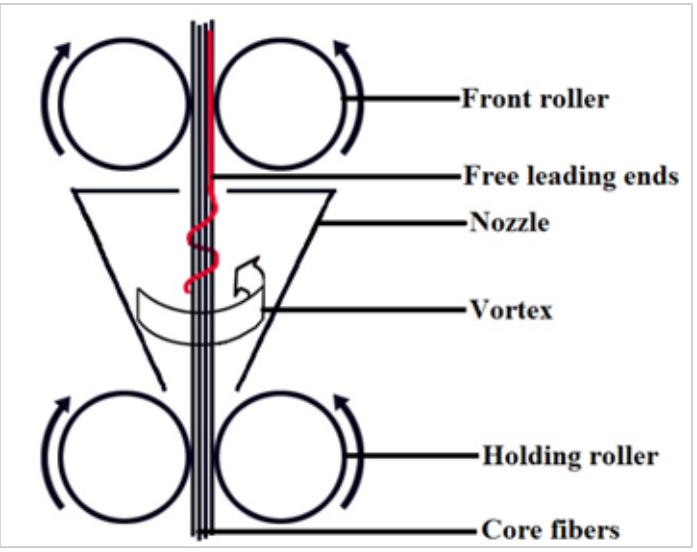

Figure 3 Fiber entanglements by the nozzle

\section{Experimental}

A modified spintester had been designed and fabricated as a prototype in our laboratory. The 370tex carded cotton roving was employed, the total draft of the classical 3 over 3 drafting roller was 23.87. There was a tension draft between the front roller and the holding roller, which could be adjusted from 1.01 to 1.05 . The air pressure was kept at $0.1 \mathrm{M} \mathrm{Pa}$ at the inlet of the nozzle. The environment of the laboratory was under standard atmosphere: the relative humidity is $65 \pm 3 \%$, the temperature is $20 \pm 2^{\circ} \mathrm{C}$. All specimens were kept in the laboratory for more than 24 hours before testing. As shown in Figure 4, some strands from the nip of the holding roller were collected and wounded on a small blackboard. For comparison, a video of the fiber strand before entering the nozzle and passing by the nozzle was recorded by Nikon digital camera D5500. Total 30 pictures which show the fiber strand clearly were captured from the video. The video was taken perpendicular to the roller stand, thus the widths of the fiber stand could be calculated from the pictures according to the ration between the width of front cot and the width of the fiber strand.

For fiber strand structure, lots of the consecutive pictures were taken by digital microscope KH-7700 and analyzed followed by the procedure adopted by Tyagi et al. ${ }^{8}$ The investigations in this paper including the classification of the fiber entanglement in the strand from holding roller and the measurement of its length and the number as well as the calculation of the widths of fiber strand before entering and passing by the nozzle. For those investigations, the thirty samples of $25-\mathrm{cm}$ lengths strands were selected randomly from the blackboard.

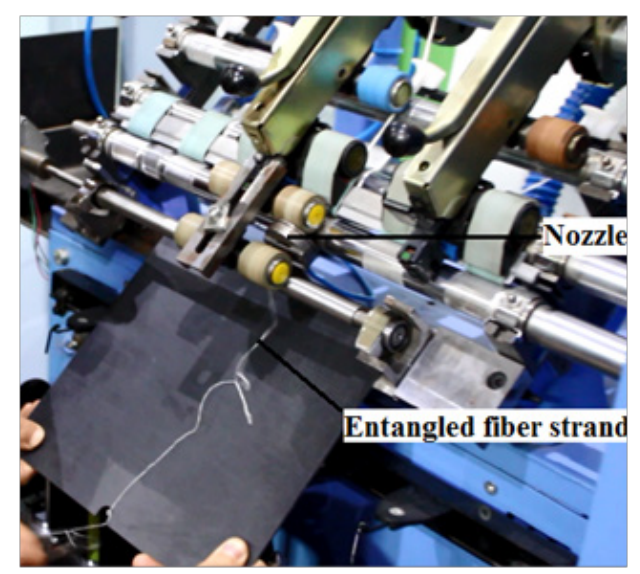

Figure 4 Collection of fiber strand

\section{Results and discussion}

Figure 5 is a picture captured from the video, which shows that the fiber strand entering the nozzle is much broader than the strand passing by the nozzle. It can be deduced that the fiber strand is compacted by the nozzle. The width of the top roller is $28 \mathrm{~mm}$. According to the ration between the widths of fiber strand and the top roller in the picture, the width of the fiber strand before entering the nozzle and passing by the nozzle can be calculated. For example, as shown in Figure 5, the width of the top roller is $30 \mathrm{~mm}$, the width of the fiber strand before entering the nozzle is $1.7 \mathrm{~mm}$, so the ration between the width of the fiber strand and the top roller in Figure 5 is 0.057 , then we can deduce that the width of fiber strand before entering the nozzle is $1.59 \mathrm{~mm}$. Total 30 pictures which show the fiber strand clearly were captured from the video. All the width of fiber strand before entering the nozzle and passing by the nozzle were calculated with aforementioned method. The average width of the strand before entering the nozzle is $1.62 \mathrm{~mm}$, the $\mathrm{CV}$ is $16.33 \%$; and the average width of the strand passing by the nozzle is $0.53 \mathrm{~mm}$, the $\mathrm{CV}$ is $19.81 \%$. Consequently, the fiber strand is significantly condensed by the nozzle.

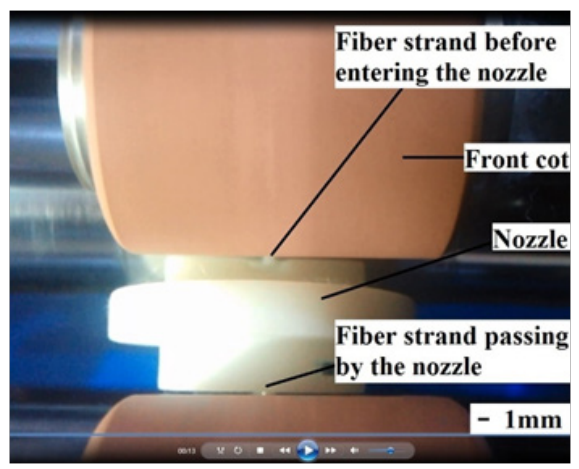

Figure 5 Width reduction of fiber strand

After careful observation and analysis on the collected strand samples, we found that the structural characteristics of the strand are similar to the classification proposed by Tyagi et al. ${ }^{8}$, as shown in Figure 6: 
a. Case (1) shows the tight wrapping. In those strands, the edge fibers are tightly wrapping the core fibers in a regular way.

b. Case (2) shows the long wrapping. In those strands, the edge fibers are wrapping the core fibers in a long way.

c. Case (3) shows the irregular wrapping. In those strands, the edge fibers are wrapping the core fibers in an irregular way.

d. Case (4) shows the unwrapped. In those strands, the edge fibers are not wrapping the core fibers, and all fibers are nearly parallel.

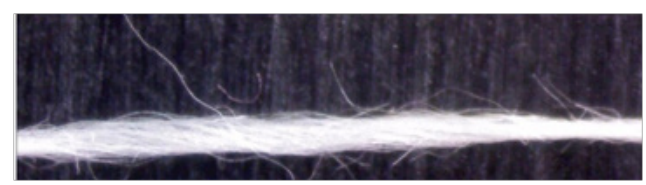

(I) Tight wrapping

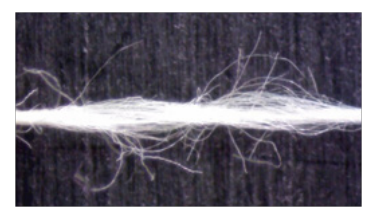

(2) Long wrapping

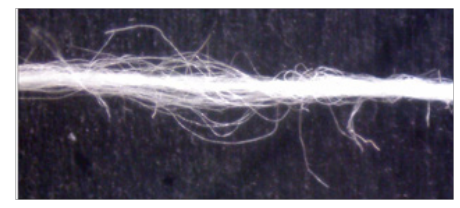

(3) Irregular wrapping

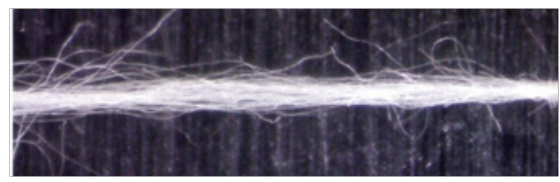

(4) Unwrapped

Figure 6 Structural characteristics of the strand passing by the nozzle

The thirty samples of $25-\mathrm{cm}$ lengths strand were consecutively observed. The proportion of different cases and the average length of each case are shown in Table 1.

From Table 1, we can see that more than $1 / 4$ strands are in wrapped structure, whatever in tight or irregular style, or long style. The results reveal how the vortex affects the edge fibers and the strand. The edge fibers could be wrapped on the core fibers and some twists are added to the strand before being twisted by ring and traveler. Those twists are in agreement with the earlier study which shows that additional twists were created for the rolling of the fiber strand in condensing zone with lattice apron. ${ }^{9}$ Consequently, a compacted strand with some twists can be created by the nozzle in a very small and closed space, and the air consumption will be less than the existed pneumatic compact system. The idea of compact spinning is to minimize the width of fiber strand before twist insertion by gathering the edge fibers, thus the flat ribbon like strand will be changed to be a thinner and round one. The test results showed that the fiber strand from the holding roller has been reached a preliminary compact effect. Compared with the rest cases, unwrapped sections are the highest in proportion, average length and $\mathrm{CV}$, while wrapped sections are the lowest in proportion, average length and CV. Unwrapped sections are quite high in the fiber strand from the holding roller, and the further spinning experiments need to be carried out to reach a better condensing effect.

Table I Statistics of the structural characteristics of fiber strand

\begin{tabular}{lcccc}
\hline & Case I & Case 2 & Case 3 & Case 4 \\
\hline Proportion (\%) & 1.93 & 8.83 & 16.69 & 72.55 \\
Average length $(\mathrm{mm})$ & 4.54 & 8.86 & 13.37 & 28.21 \\
CV\% & 12.21 & 26.89 & 31.95 & 61.18 \\
\hline
\end{tabular}

\section{Conclusion}

We have introduced a novel compact spinning. A nozzle is adopted as a condensing element and tested. The fiber strand structure characteristics show a little common to the MVS yarns, and the statistical analysis shows that more than 1/4 strands are in wrapped structure before twist insertion by ring and traveler. It can be deduced that edge fibers could be wrapped on the core fibers by the spiral airflow. The experiments showed that the fiber strand could be condensed when passing by the nozzle. When those strands are twisted by ring and traveler, a viable yarn with less hairiness may be got. Compared with the existed pneumatic compact system, the air consumption may be decreased as the airflow in this system is running in a closed and limited space.

\section{Acknowledgments}

This work is supported by grand 2017YFB0309100 from the National Key R\&D Program of China.

\section{Conflict of interest}

Authors declare there is no conflict of interest in publishing the article.

\section{References}

1. Cheng KSP, Yu C. A study of compact spun yarns. Textile Research J. 2003;73(4):345-349.

2. Lawrence CA. Advances in Yarn Spinning Technology. 1st ed. Cambridge: Woodhead Publishing Limited; 2010. 464 p.

3. Basal G, Oxenham W. Comparison of properties and structures of compact and conventional spun yarns. Textile Research J. 2006;76(7):565-575.

4. Kampen W. The advantages of condensed spinning. Melliand English. 2000;4:58-59.

5. Dou HP, Liu SR. Trajectories of fibers and analysis of yarn quality for compact spinning with pneumatic groove. $J$ Textile Institute. 2011;102(8):713-718.

6. Altas S, Kadoğlu H. Comparison of conventional ring, mechanical compact and pneumatic compact yarn spinning systems. J Engineered Fibers \& Fabrics. 2012;7(1):87-100.

7. Stadler H. Compact spinning-a new generation of ring spun yarns. Textilberichte Melliand. 1995;76(3):E29-E31.

8. Tyagi GK, Sharma D, Salhotra KR. Process-structure-property relationship of polyester-cotton MVS yarns: Part-I Influence of processing variables on the yarn structure parameters. Indian J Fibre Textile Research. 2004;29:419-428.

9. Yang X, Wang J, Yang JP. Motion analysis of fiber band in compact field of compact spinning. J Donghua University. 2006;23(1):144-147. 